\title{
LV-SY-3-3
}

\section{Two stage hepatectomy (TSH) or Associating liver partition and portal vein ligation for staged hepatectomy (ALLPS) for bilobar multiple colorectal liver metastasis}

\author{
Je Ho RYU*
}

Pusan National University School of Medicine, Yangsan, Korea

Lecture: Of patients with colorectal cancer, $30 \%$ to $50 \%$ present with liver metastasis at the time of diagnosis or develop metastasis later. Hepatectomy is the primary curative treatment option for patients with colorectal liver metastases (CRLMs).

Patients with bilateral, multiple CRLMs and a small estimated tumor free future liver remnant (FLR) present a treatment challenge. These patients may need a two-stage hepatectomy (TSH) in which portal venous ligation (PVL) or portal venous embolization (PVE) is included in the first stage to stimulate hypertrophy of the FLR before final resection. This method generates a $27 \%$ to $39 \%$ increase in FLR volume in 4 to 8 weeks. The risks in this method are tumor progression during the waiting period and insufficient hypertrophy, making resection impossible in $25 \%$ to $38 \%$ of the patients preparing to undergo TSH.

To increase liver growth rate and volume, which renders more patients resectable in a shorter time, a novel concept of liver resection, associating liver partition and portal vein ligation for staged hepatectomy (ALPPS), was described in 2011. However, in the early study $68 \%$ of the patients experienced complications and the surgical mortality rate was $12 \%$.

A recent, the only multicenter randomized controlled trial (RCT) revealed that compared with the use of TSH, the use of ALPPS for patients with CRLM resulted in a higher resection rate (92\%) without a higher 90-day mortality rate (8.3\%), a higher rate of severe complication rate $(43 \%)$ or a lower rate of negative surgical margins in the liver.

A previous meta-analysis including 9 retrospective studies showed that TSH for CRLM exhibited lower perioperative morbidity and mortality rates than ALPPS, suggesting the superiority of TSH.

On the other hand, in a recent meta-analysis in 2020 including 7 retrospective studies and 1 RCT, compared with TSH, ALPPS for advanced CRLM resulted in superior surgical efficacy with comparable perioperative mortality rate and short-term oncological outcomes, while this was at the cost of increased perioperative minor complications.

From a clinical perspective, traditional TSH has been frequently regarded as a preferred treatment modality in earlier studies, and ALPPS was reserved as an alternative, typically after failed PVE or PVL.

At present, this paradigm may have changed, as there is growing evidence indicating that the surgical benefits given by ALPPS are at least not inferior to those given by TSH according to recent studies.

ALPPS may be feasible for bilobar multiple colorectal liver metastasis. Still, long-term outcomes remain to be elucidated and therefore the role of ALPPS regarding oncological outcome remains uncertain. 